\title{
Detection of KRAS mutations in colorectal cancer with Fast COLD-PCR
}

\author{
PIETRO CAROTENUTO $^{1}$, CRISTIN ROMA $^{1}$, SALVATORE COZZOLINO ${ }^{2}$, FRANCESCA FENIZIA $^{1}$, \\ ANNA MARIA RACHIGLIO ${ }^{1}$, FABIANA TATANGELO ${ }^{3}$, ALESSIA IANNACCONE $^{1}$, \\ LUIGI BARON $^{4}$, GERARDO BOTTI ${ }^{3}$ and NICOLA NORMANNO ${ }^{5}$
}

\begin{abstract}
${ }^{1}$ Pharmacogenomic Laboratory, CROM - Centro Ricerche Oncologiche di Mercogliano, Avellino; ${ }^{2}$ Department of Structural and Functional Biology of University of Naples Federico II; ${ }^{3}$ Surgical Pathology Unit, INT Fondazione G. Pascale; ${ }^{4}$ Surgical Pathology Unit, San Leonardo Hospital; ${ }^{5}$ Cell Biology and Biotherapy Unit, INT Fondazione G. Pascale, Naples, Italy
\end{abstract}

Received July 13, 2011; Accepted September 1, 2011

DOI: 10.3892/ijo.2011.1221

\begin{abstract}
Patients with metastatic colorectal carcinoma (mCRC) carrying activating mutations of the KRAS gene do not benefit from treatment with anti-epidermal growth factor receptor (EGFR) monoclonal antibodies. Therefore, KRAS mutation testing of mCRC patients is mandatory in the clinical setting for the choice of the most appropriate therapy. Co-amplification-at-lower denaturation-temperature PCR (COLD-PCR) is a novel modification of the conventional PCR method that selectively amplifies minority alleles from a mixture of wild-type and mutant sequences irrespective of the mutation type or position within the sequence. In this study, we compared the sensitivity of a COLD-PCR method with conventional PCR/sequencing and the real-time PCR-based Therascreen kit to detect KRAS mutations. By using dilutions of KRAS mutant DNA in wild-type DNA from colon cancer cell lines with known KRAS status, we found that Fast COLD-PCR is more sensitive than the conventional PCR method, showing a sensitivity of $2.5 \%$ in detecting $\mathrm{G}>\mathrm{A}$ and $\mathrm{G}>\mathrm{T}$ mutations. The detection of $\mathrm{G}>\mathrm{C}$ transversions was not improved by either Fast COLD-PCR or Full COLD-PCR. We next analyzed by COLD-PCR, conventional PCR and Therascreen 52 formalin-fixed paraffin-embedded samples from mCRC patients. Among 36 samples with $>30 \%$ tumor cells, 8 samples were negative by conventional PCR, Therascreen and Fast COLD-PCR; 20 mutations identified by conventional PCR were confirmed by Therascreen and Fast COLD-PCR; 8 cases undetermined by conventional PCR were all confirmed to carry KRAS G $>$ A or $\mathrm{G}>\mathrm{T}$ mutations by
\end{abstract}

Correspondence to: Dr Nicola Normanno, Cell Biology and Biotherapy Unit, INT-Fondazione Pascale, Via M. Semmola, 80131 Naples, Italy

E-mail: nicnorm@yahoo.com;n.normanno@istitutotumori.na.it

Key words: KRAS, mutations, colorectal carcinoma, epidermal growth factor receptor, anti-EGFR therapy, co-amplification-atlower denaturation-temperature PCR using either Therascreen or Fast COLD-PCR. Conventional PCR was able to detect only 2 KRAS mutations among 16 samples with $<30 \%$ tumor cells (12.5\%), whereas Therascreen and Fast COLD-PCR identified 6 mutants (37.5\%). These data suggest that Fast COLD-PCR has a higher clinical sensitivity as compared with conventional PCR in detecting $\mathrm{G}>\mathrm{C}$ to $\mathrm{A}>\mathrm{T}$ changes in the KRAS gene, which represent $>90 \%$ of the mutations of this oncogene in CRC.

\section{Introduction}

KRAS is a member of the Ras gene family that encodes small G proteins with intrinsic GTPase activity (1). KRAS is a downstream component of the EGFR signalling network and links growth promoting signals from the cell surface to several, different intracellular signalling pathways, which regulate important functions for tumor progression such as proliferation, differentiation and apoptosis (1-3). Mutations of the KRAS oncogene that lead to its constitutive activation have been described in different tumor types including colorectal carcinoma (CRC) (2). KRAS mutations are an early event in colon tumorigenesis and have been detected in $\sim 30-45 \%$ of CRC (4). Up to $98 \%$ of KRAS mutations in CRC patients are found in codons 12 and 13 of exon 2, with seven mutations accounting for the majority of codons 12 and 13 alterations $(5,6)$.

A number of studies have demonstrated that patients with metastatic CRC (mCRC) carrying KRAS mutations in codon 12 and 13 do not benefit from treatment with anti-EGFR monoclonal antibodies such as cetuximab and panitumumab (7-10). Therefore, assessment of the mutational status of KRAS is mandatory in $\mathrm{mCRC}$ patients to ensure an appropriate choice of treatment (11). The most widely accessible method for KRAS testing is direct sequencing of PCR products, which is still the gold standard technique for mutation detection $(6,12)$. This method detects all mutations in amplified DNA sequences, but requires that mutant copies have a concentration that is at least $10-30 \%$ of any accompanying wild-type sequence according to the available literature $(5,6,13,14)$. For this reason, it is recommended that tissues for mutational analysis should contain at least $70 \%$ of tumor cells, and if this is not possible 
the region of the sample with the highest content of tumor cells should be isolated and used for molecular analysis. As a result, conventional PCR-based assays are limited in their ability to identify low levels of mutation-bearing tumor cells and this may greatly affect therapeutic decisions.

Co-amplification-at-lower denaturation-temperature PCR (COLD-PCR) is a novel modification of the conventional PCR method that selectively amplifies minority alleles from a mixture of wild-type and mutant sequences irrespective of the mutation type or position within the sequence (15). This method is based on the observation that there is a critical denaturation temperature $\left(\mathrm{T}_{\mathrm{c}}\right)$ for each DNA sequence, which is lower than its melting temperature $\left(\mathrm{T}_{\mathrm{m}}\right)$. DNA amplicons differing by a single nucleotide have different amplification efficiencies when PCR denaturation temperature is set to $T_{c}$.

Two forms of COLD-PCR have been described: Fast COLD-PCR and Full COLD-PCR. Fast COLD-PCR enriches $\mathrm{G}>\mathrm{C}$ to $\mathrm{A}>\mathrm{T}$ mutations that slightly, but predictably lower the $\mathrm{T}_{\mathrm{m}}$ of the PCR amplicon, by using a $\mathrm{T}_{\mathrm{c}}$ that favors PCR amplification of the mutant allele. Full COLD-PCR can theoretically enhance detection of any type of mutation via conditions which promote annealing of WT:mutant pairs and selective denaturation of these heteroduplexes at an empirically determined $\mathrm{T}_{\mathrm{c}}$. Fast COLD-PCR has the advantage of being more rapid than Full COLD-PCR (1-2 h compared to 5-8 h of PCR cycling) and is also easier to troubleshoot and implement. Fast COLD-PCR is ideal for KRAS codon 12 and 13 mutational analysis because $>90 \%$ of the mutations are either $\mathrm{G}>\mathrm{A}$ or G>T changes (G12D, G12V, G12 S, G12C, G13D), whereas the remaining common mutations are $\mathrm{T}_{\mathrm{m}}$-neutral $\mathrm{G}>\mathrm{C}$ changes (G12R and G12A).

In this study, we compared a newly developed COLD-PCR method with a conventional PCR/sequencing method, routinely used in our laboratory for the detection of KRAS mutations, and with the real-time PCR-based Therascreen kit, a high sensitive commercial method approved for in vitro clinical diagnostics $(13,14)$.

\section{Materials and methods}

Samples. Formalin-fixed paraffin-embedded (FFPE) tissues of 52 patients were obtained from the institutional Tissue Bank following approval of the Institutional Review Board. The tumor cell content of each sample was assessed by a referral pathologist (G.B.) on a slide stained with hematoxylin and eosin. For tumor samples with a percentage of tumor cells $<70 \%$, a macrodissection of the specimen was performed if possible, in order to increase the relative content of tumor cells.

The following cell lines obtained from ATCC (American Type Culture Collection, Rockville, MD) were used: the colorectal carcinoma cell lines HT29 (wild-type for KRAS), LS174T (bearing the KRAS mutation 35G $>$ A), SW620 (35G>T), HCT116 (38G>A), SW1116 (35G>C) and SW1463 (34G>T), the lung carcinoma cell line A549 (34G>A). Thyroid carcinoma CaL-62 cells (34G $>$ C) were kindly provided by Professor M. Santoro (University Federico II, Naples, Italy).

DNA extraction. Genomic DNA was extracted from two $20-\mu \mathrm{m}$ FFPE sections using the QIAamp ${ }^{\circledR}$ DNA FFPE Tissue kit (Qiagen) and the QIAcube apparatus (Qiagen), according to manufacturer's instructions. Genomic DNA from cancer cell lines was isolated using the DNeasy blood and tissue kit (Qiagen).

Samples of isolated genomic DNA were analysed by $0.8 \%$ agarose gel electrophoresis to evaluate the DNA quality. The DNA quantity was assessed by using the Nanovue (GE Healthcare) and the purity was evaluated by calculating the $260 / 280$ ratio.

KRAS mutational analysis by conventional PCR. The genomic region harbouring mutational sites was amplified using the following primers to obtain a 201-bp amplicon: Forward, 5'-TGTAAAACGACGGCCAGTTGTGACATGTTCTAATA TAGTCACATT-3'; Reverse: 5'-CAGGAAACAGCTATGA $C C A C C A G T A A T A T G C A T A T T A A A A C A A G A-3 '$ '. The nucleotides presented in italic type correspond to M13 consensus sequences and were used also for cycle sequencing with M13 consensus primers. Oligonucleotides primers were purchased from PRIMM (Milan, Italy).

PCR was performed in $50 \mu$ reaction volume containing 1X AmpliTaq ${ }^{\circledR}$ Gold DNA polymerase buffer (Applied Biosystems, Foster City , USA); $2.5 \mathrm{mM} \mathrm{MgCl}_{2} ; 0.02 \mathrm{mM}$ each deoxynucleotide; $0.2 \mu \mathrm{M}$ each primer; 2.5 units AmpliTaq ${ }^{\circledR}$ Gold DNA Polymerase (Applied Biosystems) and $80 \mathrm{ng}$ of DNA template. PCR reactions were performed by incubating the samples at $95^{\circ} \mathrm{C}$ for $10 \mathrm{~min}$, followed by 40 cycles of $95^{\circ} \mathrm{C}$ for $30 \mathrm{sec}, 58^{\circ} \mathrm{C}$ for $30 \mathrm{sec}$ and $72^{\circ} \mathrm{C}$ for $1 \mathrm{~min}$. The final extension step was performed for $10 \mathrm{~min}$ at $72^{\circ} \mathrm{C}$ and the samples were then chilled to $4^{\circ} \mathrm{C}$. PCR reactions were run in a Veriti ${ }^{\circledR}$ Thermocycler (Applied Biosystems). The PCR products were electrophoresed in an agarose gel to confirm successful amplification.

Identification of the critical denaturation temperature. We applied the COLD-PCR method for detecting KRAS mutations based on the methodology described in the literature (15), with several modifications. We determined $\mathrm{T}_{\mathrm{c}}$ experimentally for each amplicon using mutant DNAs obtained from LS174T, SW620, HCT116, SW1116 and SW1463, A549 and CaL-62 cells lines, bearing the seven common mutations in codon 12 and 13 of KRAS gene. A set of COLD-PCR reactions at graded temperatures below the $\mathrm{T}_{\mathrm{m}}$ were performed, to identify the optimal critical denaturation temperature, $\mathrm{T}_{\mathrm{c}}$, and direct sequencing of PCR product was used to determine the degree to which the mutant allele was enriched. We determined the $\mathrm{T}_{c}$ empirically by progressively lowering the denaturation temperature from $84^{\circ} \mathrm{C} \rightarrow 82^{\circ} \mathrm{C} \rightarrow 81^{\circ} \mathrm{C} \rightarrow 80^{\circ} \mathrm{C} \rightarrow 79^{\circ} \mathrm{C}$.

The denaturation temperature that reproducibly produces robust PCR products combined with substantial enrichment of the mutations was selected as the $T_{c}$. By following this experimental procedure for all mutations, we set the reduced denaturation temperature of the COLD-PCR reaction at $82^{\circ} \mathrm{C}$.

COLD-PCRfor KRAS mutation detection. For the COLD-PCR protocols we used the same primers and reagents used in the conventional PCR assay. Fast COLD-PCR started with 25 cycles of conventional PCR amplification for an initial build up of all amplicons, followed by 30 COLD-PCR cycles to selectively enrich for mutant sequences. The initial conventional 
Fast COLD-PCR

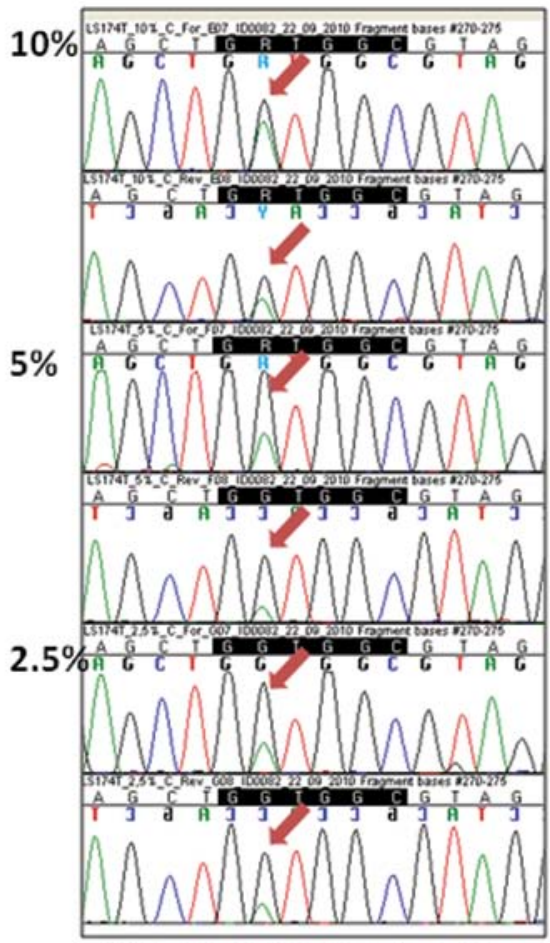

$1 \%$

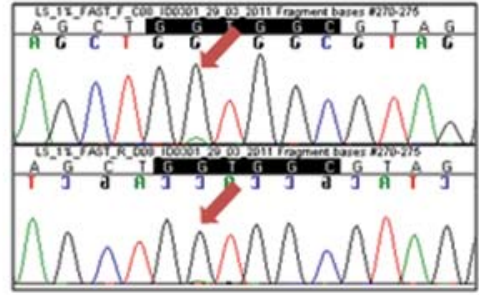

CLASSIC PCR-SEQ
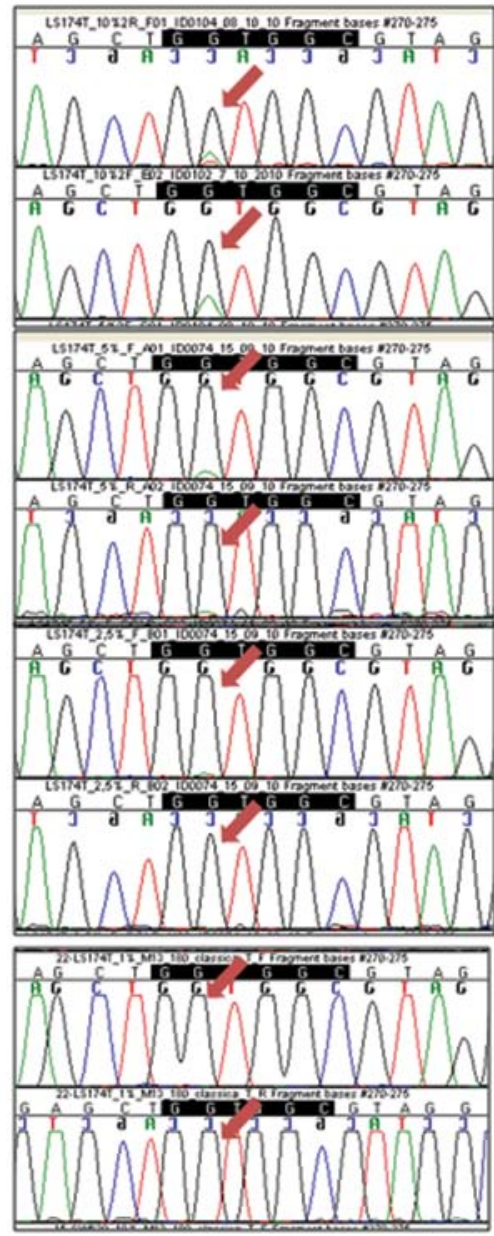

Figure 1. Sensitivity of conventional PCR/sequencing and Fast COLD-PCR methods. DNA from LS174T cells that carry the G35A KRAS mutation was diluted with wild-type DNA obtained from HT-29 colon carcinoma cells in proportions of 10, 5, 2.5, $1 \%$ mutant DNA. Sequencing chromatograms of forward reverse sequences are reported. Arrows show the peak corresponding to the mutated nucleotide.

PCR cycling conditions are summarized as follows: $95^{\circ} \mathrm{C}$ for $10 \mathrm{~min} ; 25$ cycles at $95^{\circ} \mathrm{C}$ for $30 \mathrm{sec}, 58^{\circ} \mathrm{C}$ for $30 \mathrm{sec}$, and $72^{\circ} \mathrm{C}$ for $1 \mathrm{~min}$. Then 30 cycles of COLD-PCR were performed at $82^{\circ} \mathrm{C}$ for $20 \mathrm{sec}, 58^{\circ} \mathrm{C}$ for $30 \mathrm{sec}, 72^{\circ} \mathrm{C}$ for $1 \mathrm{~min}$ and one step at $72^{\circ} \mathrm{C}$ for $10 \mathrm{~min}$.

The reaction protocol for Full COLD-PCR started with 25 cycles of the conventional PCR amplification as above described, followed by 30 COLD-PCR cycles performed at $95^{\circ} \mathrm{C}$ for $30 \mathrm{sec}, 70^{\circ} \mathrm{C}$ for $4 \mathrm{~min}, 82^{\circ} \mathrm{C}$ for $20 \mathrm{sec}, 58^{\circ} \mathrm{C}$ for $30 \mathrm{sec}, 72^{\circ} \mathrm{C}$ for $1 \mathrm{~min}$ and one step at $72^{\circ} \mathrm{C}$ for $10 \mathrm{~min}$. PCR reactions were performed on Veriti ${ }^{\circledR}$ Thermocycler (Applied Biosystems). The PCR products were electrophoresed in agarose gels to confirm successful amplification prior to sequencing as described above.

Sanger sequencing. Following PCR, amplification products were purified using the pre-sequencing Kit ExoSap-IT reagent (Amersham Biosciences). Sequencing reactions were performed with the BigDye ${ }^{\circledR}$ Terminator Cycle Sequencing Kit v1.1 (Applied Biosystems) chemistry using both M13-F and M13-R sequencing primers to obtain forward and reverse sequences. Cycle Sequencing reactions were cleaned up using BigDye
Terminator purification kit (Applied Biosystems). Purified sequencing reactions were analyzed using both the Applied Biosystems 3130 Genetic Analyzer and the Applied Biosystems 3500 Genetic Analyzer. Similar results were obtained with the two different apparatus. The sequence data were analysed using the Sequencer software Ver. 4.8 (Gene Codes Corporation, Ann Arbor, USA) or SeqScape ${ }^{\circledR}$ Software v2.7 (Applied Biosystems) to identify mutations and to assign genotypes to individual DNA samples.

The identified DNA changes were compared to reference sequence of KRAS (GeneBank accession no. NM_004449.3). Sequence results were scored by visual inspection of the chromatograms, performed by three independent analysts (NN, PC, CR). A mutation was called when the three independent observers agreed.

Real-time PCR with Therascreen kit. Mutational analysis was performed by using the Therascreen KRAS mutations kit (DxS Ltd., Manchester, UK) that identifies the seven most frequent somatic mutations located in codons 12 and 13 (35G $>\mathrm{A}$; $35 \mathrm{G}>\mathrm{C}$; 35G $>\mathrm{T} ; 34 \mathrm{G}>\mathrm{A} ; 34 \mathrm{G}>\mathrm{C} ; 34 \mathrm{G}>\mathrm{T}$ and $38 \mathrm{G}>\mathrm{A})$. Samples were analysed according to the manufacturer's protocol and 
Table I. Comparison of conventional PCR/sequencing, Therascreen and fast COLD-PCR methods in mCRC samples.

\begin{tabular}{lcccc}
\hline & & \multicolumn{3}{c}{ Mutations detected } \\
\cline { 3 - 5 } Sample group & Tumor cell content $(\%)$ & PCR-SEQ $^{\mathrm{a}}$ & Therascreen $^{\text {Fast COLD-PCR }}$ \\
\hline WT $(\mathrm{N}=8)$ & $>30$ & 0 & 0 & 0 \\
Mutant $(\mathrm{N}=20)$ & $>30$ & 20 & 20 & 20 \\
Undetermined $(\mathrm{N}=8)$ & $>30$ & 0 & 8 & 8 \\
Low tumor cell content $(\mathrm{N}=16)$ & $\leq 30$ & 2 & 6 & 6 \\
\hline
\end{tabular}

${ }^{\mathrm{a} C o n v e n t i o n a l ~ P C R ~ f o l l o w e d ~ b y ~ S a n g e r ~ s e q u e n c i n g . ~}$

by using an ABI PRISM 7900HT real-time PCR system (Applied Biosystems). Sample $\Delta \mathrm{Ct}$ values are calculated as the difference between the mutation assay $\mathrm{Ct}$ and control assay $\mathrm{Ct}$ from the same sample. Threshold $\Delta \mathrm{Ct}$ for each mutation are indicated by the manufacturer, and correspond to the $\Delta \mathrm{Ct}$ for $1 \%$ of mutant DNA. Above this value, the sample may contain $<1 \%$ mutation (beyond the limit of the assays) and the sample is considered mutation negative. The $\Delta \mathrm{Ct}$ cut-off values were as follows: $35 \mathrm{G}>\mathrm{A}(\mathrm{G} 12 \mathrm{D})$ cut-off $8 ; 35 \mathrm{G}>\mathrm{C}(\mathrm{G} 12 \mathrm{~A})$ cut-off $6.5 ; 35 \mathrm{G}>\mathrm{T}(\mathrm{G} 12 \mathrm{~V})$ cut-off 6.5 ; $34 \mathrm{G}>\mathrm{A}(\mathrm{G} 12 \mathrm{~S})$ cut-off $9 ; 34 \mathrm{G}>\mathrm{C}(\mathrm{G} 12 \mathrm{R})$ cut-off $8 ; 34 \mathrm{G}>\mathrm{T}$ (G12C) cut-off 7 and 38G $>$ A (G13D) cut-off 9 .

Statistical analysis. The correlation between KRAS mutation frequency data obtained using PCR/sequencing, COLD-PCR or the Therascreen kit, and tumor cell percentage was assessed using Fisher's exact test. All tests were performed two-sided at a significance level of 0.05 . The statistical tests were performed using SPSS version 12.0 (SPSS Inc, Chicago, IL, USA).

\section{Results}

Sensitivity of KRAS mutation testing procedures. The sensitivity of conventional and COLD-PCR assays was determined as previously described (14). Genomic DNA was isolated from cancer cell lines carrying the seven most frequent mutations of the KRAS gene (HCT-116, SW620, SW1116, LS174T, A549, SW1463 and CaL-62). Mutant DNA was diluted with wild-type DNA obtained from HT-29 colon carcinoma cells in proportions of $100,80,50,40,20,10,5,2.5,1 \%$. The DNA mixtures were amplified by PCR and sequences were analyzed to identify the presence of the different mutations. The lowest level of detection for conventional PCR was 10\% mutant DNA in a background of wild-type DNA as found in three independent experiments, whereas the Fast COLD-PCR assay was found to detect up to $2.5 \%$ of mutant DNA. Representative results are shown in Fig. 1. The Fast COLD-PCR method was more sensitive than conventional PCR for $\mathrm{G}>\mathrm{A}$ and $\mathrm{G}>\mathrm{T}$ mutations, while the sensitivity was not improved for $\mathrm{G}>\mathrm{C}$ transversions. Similar results were obtained with the Full COLD-PCR protocols that unexpectedly did not improve the detection of the $\mathrm{G}>\mathrm{C}$ transversions (data not shown).

Comparison of KRAS mutation detection using conventional PCR, COLD-PCR and Therascreen. We further examined the potential enhancement of KRAS mutation detection by COLD-PCR over conventional PCR in clinical samples. We have previously demonstrated that the Therascreen kit, which has a sensitivity limit of $1 \%$, shows an higher analytical sensitivity as compared with conventional PCR followed by direct sequencing only in specimens that contain $30 \%$ or less of tumor cells (14). Therefore, we selected 52 FFPE samples from colorectal carcinoma patients with different levels of tumor cells: 36 with $>30 \%$ tumor cells and 16 with $<30 \%$ tumor cells. For the samples with $>30 \%$ tumor cells, we selected 8 samples that were wild-type by conventional PCR-sequencing, 20 cases that were mutant according to conventional PCR, and 8 cases in which this method did not allow to assess the mutational status. In particular, the analysis of sequencing chromatograms of these latter samples showed the presence of low-intensity peaks within codons 12 and 13 of the KRAS gene that might be suggestive of potential mutations. However, the low intensity of the signals that in most cases were not consistently represented in all the chromatograms did not allow to define the mutational status.

The samples were tested with conventional PCR-sequencing, Fast COLD-PCR-sequencing and the Therascreen kit (Table I and Fig. 2). The 8 samples that were negative by conventional PCR (WT group) were negative also following analysis with the Therascreen or with Fast COLD-PCR. Analogously, the 20 mutations identified by conventional PCR (mutant group) were confirmed by Therascreen and COLD-PCR. Interestingly, in one sample the use of COLD-PCR led to amplification of the mutant allele alone and the sample appeared as a homozygous mutant (Fig. 2). The 8 cases undetermined by conventional PCR (undetermined group) were all confirmed to carry mutant KRAS alleles by using either the Therascreen or Fast COLD-PCR. All these undetermined cases were G $>A$ or $\mathrm{G}>\mathrm{T}$ mutations. Among the samples with $<30 \%$ tumor cells (low tumor cell content group), conventional PCR was able to detect only 2 KRAS mutations (12.5\%). In contrast, both Therascreen and Fast COLD-PCR identified 4 additional mutations, for a total of 6/16 mutants (37.5\%). Importantly, in these samples Therascreen and Fast COLD-PCR found the same mutations. The difference in the mutation rate between these latter methods and conventional PCR was statistically significant ( $<<0.0001$, Fisher's exact test). Intriguingly, one of the mutations that was not detected by conventional PCR was a $\mathrm{G}>\mathrm{C}$ that was enhanced by Fast COLD-PCR (Fig. 2). The use of Full COLD-PCR did not produce a further enhancement of the mutant signal (data not shown). 


\section{CLASSIC PCR-SEQ}
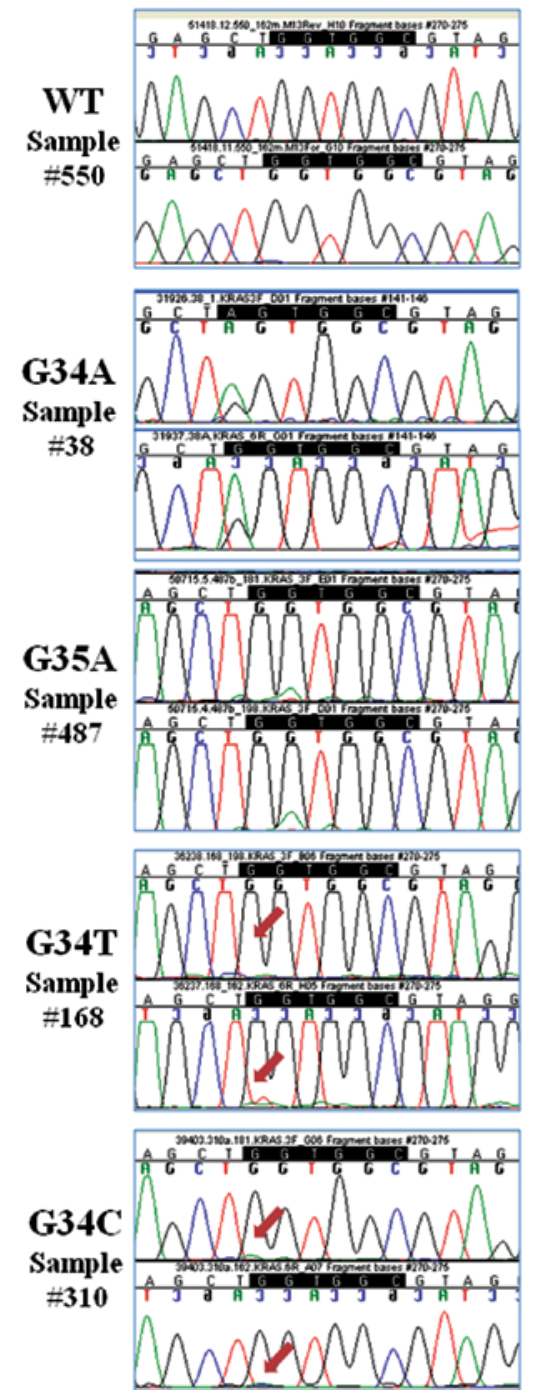

Fast COLD-PCR
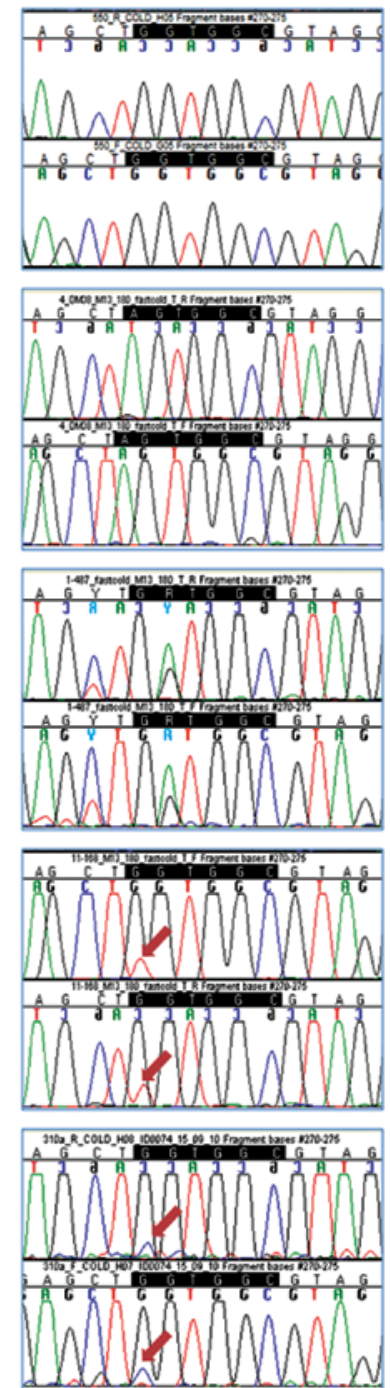

\section{THERASCREEN}
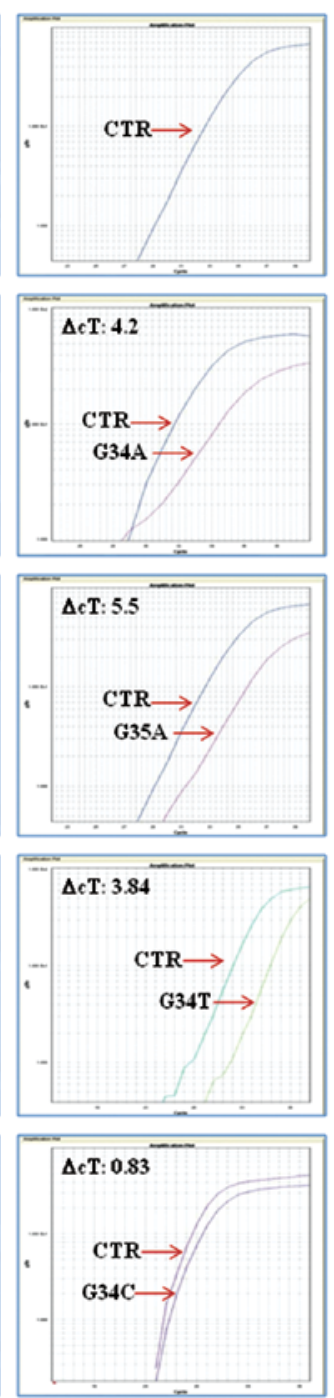

Figure 2. Representative results of KRAS genotyping using PCR/sequencing, COLD-PCR and Therascreen. A wild-type (sample 550) and four mutated samples are shown. Samples 168 and 310 belong to the group with undeterminated results using PCR/sequencing. Arrows in the chromatograms indicate the peaks potentially associated with a KRAS mutation. CTR, control assay.

\section{Discussion}

Assessment of KRAS mutational status is mandatory for a correct plan of the therapeutic strategy in mCRC patients. A number of different factors might affect the sensitivity and the specificity of the mutational analysis, including a low tumor cell content; the presence of DNA fragmentation and, more generally, of a poor quality DNA; tumor heterogeneity with presence of mutant and wild-type tumor cell clones within the same specimen. Therefore, mutational analysis might result in false positive as well as in false negative results. Patients with a false positive KRAS mutation will be deprived of the possibility to benefit of treatment with anti-EGFR agents. False negative results that do not identify KRAS mutations might also be deleterious for patients. In fact, anti-EGFR agent are not active in patients carrying a mutant KRAS. In addition, recent findings suggest that administration of an anti-EGFR monoclonal antibody in combination with a regimen containing oxaliplatin to patients with a KRAS mutant tumor might significantly reduce progression-free survival $(16,17)$.

$\mathrm{PCR} /$ sequencing is the most widely used method for mutational analysis and it still represents the gold standard for this type of analysis. We have previously demonstrated that PCR/ sequencing can reach up to $10 \%$ sensitivity and that it allows to define the mutational status of KRAS in FFPE tissues from mCRC patients in the majority of the cases (14). However, we also found that this method has not sufficient analytical sensitivity in those samples that have a tumor cell content of $30 \%$ or lower. In addition, in $2 \%$ of cases with a higher tumor cell content, PCR/sequencing does not allow defining the mutational status with certainty, and in these latter samples more sensitive techniques, such as the real-time PCR-based Therascreen kit, are required. In this respect, this study demonstrates for the first time that COLD-PCR is equivalent to Therascreen in clinical samples. In fact, we found that in samples with a low tumor cell content $(\leq 30 \%)$ and in cases in 
which conventional PCR followed by Sanger sequencing did not result in a conclusive diagnosis, Fast COLD-PCR followed by sequencing has a similar clinical sensitivity to Therascreen being able to identify the same mutations. Importantly, neither method resulted in false positive findings.

Our results are in agreement with previous studies suggesting that COLD-PCR might enhance the detection of KRAS mutations in CRC. Li et al (15) showed that COLD-PCR can enhance the sensitivity of both Sanger sequencing and pyrosequencing. In particular, full COLD-PCR was reported to enrich the different KRAS mutations 3-12-fold, depending on the mutation type, whereas Fast COLD-PCR increased the sensitivity of 10-100-fold for those mutations that result in a decrease of the $\mathrm{T}_{\mathrm{m}}$. However, a limited number of clinical samples were examined in this study and the enhancement of COLD-PCR for the seven most common KRAS mutations was not shown. More recently, Zuo et al (18) used a full COLD-PCR protocol to increase the ability of pyrosequencing to detect KRAS mutations in 30 FFPE CRC tissues. This approach resulted in an $\sim 2$-fold enhancement that was observed only in 9/20 mutant samples. Taken together, these results suggest that although full COLD-PCR has the theoretical advantage to enhance all the mutations, the level of enhancement is slight and is not observed for all the samples. Since $>90 \%$ of the KRAS mutations are $\mathrm{G}>\mathrm{A}$ or $\mathrm{G}>\mathrm{T}$ changes, Fast COLD-PCR is ideal for KRAS codon 12 and 13 mutational analysis. Indeed, Pritchard et al (19) found that Fast COLD-PCR enhanced the diagnostic accuracy of melting curve analysis in CRC. In particular, this method was able to increase the sensitivity for the $\mathrm{G}>\mathrm{C}$ to $\mathrm{A}>\mathrm{T}$ changes up to $1 \%$, without affecting the sensitivity of the assay for the $\mathrm{T}_{\mathrm{m}}$-neutral $\mathrm{G}>\mathrm{C}$ changes. Melting curve analysis is a screening method that is often used to detect rare mutations and that needs to be confirmed by sequencing of the PCR product due to the possibility of false positive results. Since KRAS mutations occur in $\sim 40 \%$ of CRC patients, the use of a screening method is of little utility in this disease. A nested PCR approach including a conventional PCR round plus a COLD-PCR round has also been shown to result in high sensitive detection of KRAS mutations in lung carcinoma patients by using either melting analysis or Sanger sequencing (20). The use of nested PCR in clinical diagnostics should be limited for the possibility of contaminations from post-PCR products that can lead to false positive results. In addition, this approach led to a $2.5 \%$ sensitivity of Sanger sequencing that was reached in our study with a single round of COLD-PCR.

As compared with the Therascreen, Fast COLD-PCR has the advantages that it does not require additional instruments and there are no additional costs as compared with Sanger sequencing. In particular, the cost of the analysis per patient is at least 5 times lower for COLD-PCR/sequencing as compared with Therascreen ( 40 vs. $200 €$ ). In contrast, the limit of Fast COLD-PCR is that it does not enhance mutations that do not affect the $\mathrm{T}_{\mathrm{m}}$. However, in agreement with previous findings, we observed enhancement of the sequencing signal in a sample carrying a G34>C mutation (Fig. 2).

One important question to address is whether highly sensitive techniques should be used for all samples or reserved to specific subsets (i.e. low percentage of tumor cells or inconclusive cases). In this regard, two different studies have demonstrated that there is no statistically significant difference in the ability to detect mutations between Therascreen and $\mathrm{PCR} /$ sequencing in samples that contain $>30 \%$ of tumor cells $(14,21)$. In particular, Therascreen detected KRAS mutations in only $1 / 95$ cases that were clearly negative by PCR/sequencing and had a tumor cell content $>30 \%$. Since the analysis with COLD-PCR is time-consuming, we believe that it should be reserved to selected cases. In addition, the use of COLD-PCR for samples that have high levels of mutant DNA might lead to lack of amplification of the wild-type allele and to a classification of the sample as homozygous for the KRAS mutation (Fig. 2). Homozygous KRAS mutations are a rare event in CRC patients. Although we have no information regarding the outcome of patients with homozygous KRAS mutations, this misclassification should be avoided. In conclusion, the Fast COLD-PCR-sequencing method that we developed is a simple, highly sensitive and cost-effective technique to detect KRAS mutations in selected CRC samples.

\section{Acknowledgements}

We thank Dr Rosella Petraroli for helpful discussion regarding the technical data.

\section{References}

1. Malumbres $\mathrm{M}$ and Barbacid M: RAS oncogenes: the first 30 years. Nat Rev Cancer 3: 459-465, 2003.

2. Schubbert S, Shannon K and Bollag G: Hyperactive Ras in developmental disorders and cancer. Nat Rev Cancer 7: 295-308, 2007.

3. Normanno N, De Luca A, Bianco C, et al: Epidermal growth factor receptor (EGFR) signaling in cancer. Gene 366: 2-16, 2006.

4. Fearon ER and Vogelstein B: A genetic model for colorectal tumorigenesis. Cell 61: 759-767, 1990.

5. Normanno N, Tejpar S, Morgillo F, De Luca A, Van Cutsem E and Ciardiello F: Implications for KRAS status and EGFRtargeted therapies in metastatic CRC. Nat Rev Clin Oncol 6: 519-527, 2009.

6. van Krieken JH, Jung A, Kirchner T, et al: KRAS mutation testing for predicting response to anti-EGFR therapy for colorectal carcinoma: proposal for an European quality assurance program. Virchows Arch 454: 233-235, 2009.

7. Amado RG, Wolf M, Peeters M, et al: Wild-type KRAS is required for panitumumab efficacy in patients with metastatic colorectal cancer. J Clin Oncol 26: 1626-1634, 2008.

8. Karapetis CS, Khambata-Ford S, Jonker DJ, et al: K-ras mutations and benefit from cetuximab in advanced colorectal cancer. $\mathrm{N}$ Engl J Med 359: 1757-1765, 2008.

9. Bokemeyer C, Bondarenko I, Makhson A, et al: Fluorouracil, leucovorin, and oxaliplatin with and without cetuximab in the first-line treatment of metastatic colorectal cancer. J Clin Oncol 27: 663-671, 2009.

10. Van Cutsem E, Kohne CH, Hitre E, et al: Cetuximab and chemotherapy as initial treatment for metastatic colorectal cancer. N Engl J Med 360: 1408-1417, 2009.

11. Allegra CJ, Jessup JM, Somerfield MR, et al: American Society of Clinical Oncology provisional clinical opinion: testing for KRAS gene mutations in patients with metastatic colorectal carcinoma to predict response to anti-epidermal growth factor receptor monoclonal antibody therapy. J Clin Oncol 27: 2091-2096, 2009.

12. Jimeno A, Messersmith WA, Hirsch FR, Franklin WA and Eckhardt SG: KRAS mutations and sensitivity to epidermal growth factor receptor inhibitors in colorectal cancer: practical application of patient selection. J Clin Oncol 27: 1130-1136, 2009.

13. Carotenuto P, Roma C, Rachiglio AM and Normanno N: Letter to the editor: reply to Kobunai et al. Pharmacogenomics 12: 309-310, 2011.

14. Carotenuto P, Roma C, Rachiglio AM, et al: Detection of KRAS mutations in colorectal carcinoma patients with an integrated PCR/sequencing and real-time PCR approach. Pharmacogenomics 11: 1169-1179, 2010. 
15. Li J, Wang L, Mamon $\mathrm{H}$, Kulke MH, Berbeco $\mathrm{R}$ and Makrigiorgos GM: Replacing PCR with COLD-PCR enriches variant DNA sequences and redefines the sensitivity of genetic testing. Nat Med 14: 579-584, 2008.

16. Bokemeyer C, Bondarenko I, Hartmann JT, et al: Efficacy according to biomarker status of cetuximab plus FOLFOX-4 as first-line treatment for metastatic colorectal cancer: the OPUS study. Ann Oncol 22: 1535-1546, 2011.

17. Douillard JY, Siena S, Cassidy J, et al: Randomized, phase III trial of panitumumab with infusional fluorouracil, leucovorin, and oxaliplatin (FOLFOX4) versus FOLFOX4 alone as firstline treatment in patients with previously untreated metastatic colorectal cancer: the PRIME study. J Clin Oncol 28: 4697-4705, 2010 .
18. Zuo Z, Chen SS, Chandra PK, et al: Application of COLD-PCR for improved detection of KRAS mutations in clinical samples. Mod Pathol 22: 1023-1031, 2009.

19. Pritchard C, Akagi L, Reddy P, Joseph L and Tait J: COLD-PCR enhanced melting curve analysis improves diagnostic accuracy for KRAS mutations in colorectal carcinoma. BMC Clin Pathol 10: 6, 2010.

20. Song C, Milbury CA, Li J, Liu P, Zhao M and Makrigiorgos GM: Rapid and sensitive detection of KRAS mutation after fastCOLD-PCR enrichment and high-resolution melting analysis. Diagn Mol Pathol 20: 81-89, 2011.

21. Tol J, Dijkstra JR, Vink-Borger ME, et al: High sensitivity of both sequencing and real-time PCR analysis of KRAS mutations in colorectal cancer tissue. J Cell Mol Med 14: 2122-2131, 2010. 